\title{
Forkhead-box series expression network is associated with outcome of clear-cell renal cell carcinoma
}

\author{
ZHONGWEI JIA $^{1,2^{*}}$, FANGNING WAN ${ }^{1,2^{*}}$, YAO ZHU ${ }^{1,2}$, GUOHAI SHI ${ }^{1,2}$, \\ HAILIANG ZHANG ${ }^{1,2}$, BO DAI ${ }^{1,2}$ and DINGWEI YE ${ }^{1,2}$ \\ ${ }^{1}$ Department of Urology, Fudan University Shanghai Cancer Center; ${ }^{2}$ Department of Oncology, \\ Fudan University Shanghai Medical College, Shanghai 200032, P.R. China
}

Received March 2, 2016; Accepted July 17, 2017

DOI: $10.3892 / \mathrm{ol} .2018 .8405$

\begin{abstract}
Previous studies have demonstrated that several members of the Forkhead-box (FOX) family of genes are associated with tumor progression and metastasis. The objective of the current study was to screen candidate FOX family genes identified from analysis of molecular networks in clear cell renal cell carcinoma (ccRCC). The expression of FOX family genes as well as FOX family-associated genes was examined, and Kaplan-Meier survival analysis was performed in The Cancer Genome Atlas (TCGA) cohort $(n=525)$. Patient characteristics, including sex, age, tumor diameter, laterality, tumor-node-metastasis, tumor grade, stage, white blood cell count, platelet count, the levels of hemoglobin, overall survival (OS) and disease-free survival (DFS), were collected for univariate and multivariate Cox proportional hazards ratio analyses. A total of seven candidate FOX family genes were selected from the TCGA database subsequent to univariate and multivariate Cox proportional hazards ratio analyses. FOXA1, FOXA2, FOXD1, FOXD4L2, FOXK2 and FOXL1 were associated with poor OS time, while FOXA1, FOXA2, $F O X D 1$ and $F O X K 2$ were associated with poor DFS time $(\mathrm{P}<0.05)$. FOXN2 was associated with favorable outcomes for overall and disease-free survival $(\mathrm{P}<0.05)$. In the gene cluster network analysis, the expression of FOX family-associated genes, including nuclear receptor coactivator (NCOA)1, NADH-ubiquinone oxidoreductase flavoprotein 3 (NDUFV3), phosphatidylserine decarboxylase (PISD) and pyruvate kinase liver and red blood cell $(P K L R)$, were independent prognostic factors for OS in patients with ccRCC. Results of the present
\end{abstract}

Correspondence to: Dr Bo Dai or Dr Dingwei Ye, Department of Urology, Fudan University Shanghai Cancer Center, 270 Dongan Road, Shanghai 200032, P.R. China

E-mail: bodai1978@126.com

E-mail: dwyeli@163.com

*Contributed equally

Key words: clear cell renal cell carcinoma, Forkhead-box family gene, prognosis study revealed that the expression of FOX family genes, including FOXA1, FOXA2, FOXD1, FOXD4L2, FOXK2 and FOXL1, and FOX family-associated genes, including NCOA1, NDUFV3, PISD and PKLR, are independent prognostic factors for patients with ccRCC.

\section{Introduction}

Renal cell carcinoma (RCC), which accounts for 2-3\% of all adult malignancies, is a relatively common malignancy with an incidence rate that is increasing at a rate of $2 \%$ each year (1). Clear cell renal cell carcinoma (ccRCC), which accounts for $\sim 90 \%$ of RCC cases (2), is the most common histological subtype of RCC and exhibits a 5 -year disease-specific survival rate of 50-69\% (3). RCC is notoriously refractory to radiation therapy and standard chemotherapy. If detected at an early stage, ccRCC can be cured by surgery. However, $25 \%$ of patients with RCC are identified with lymph node metastasis or distant metastasis at first diagnosis, and $30-40 \%$ of patients experience recurrence or metastasis even following surgery (4). Currently, the primary prognostic index for ccRCC is the Fuhrman nuclear grade and disease staging at the time of surgery (5). Thus, it is important to develop new biomarkers to screen out high-risk patients for additional appropriate postoperative therapy and surveillance.

Forkhead-box (FOX) family proteins are involved in the regulation of cell growth and differentiation as well as embryogenesis and tissue development. These proteins are characterized by a conserved FOX domain and extra-FOX protein-protein interaction domains (6). The FOX domain is $\sim 100$ amino acids in length and is involved in DNA binding (6-8). The extra-FOX regions are involved in interactions with transcriptional activators, transcriptional repressors or DNA repair complexes $(6,7)$. Previous studies have demonstrated an association between the expression of FOX family genes and the prognosis of different types of cancer, including lung cancer, basal cell carcinoma, esophageal cancer, pancreatic cancer, rhabdomyosarcoma, acute myeloblastic leukemia and acute lymphocytic leukemia (9). However, the role of FOX family genes in ccRCC has not been described.

The present study examined the expression of FOX family genes in 525 ccRCC cases from The Cancer 
Genome Atlas (TCGA) database with the aim of potentially identifying a prognostic marker for ccRCC. The associations between FOX family-related gene expression and clinicopathological characteristics were also investigated.

\section{Materials and methods}

Patients and data. The expression levels of FOX family genes, FOX family-related genes, and associated clinical data were downloaded from the TCGA data portal, which is available from the Cancer Genomics Browser of the University of California Santa Cruz (https://genome-cancer.ucsc.edu/). A total of 51 gene members of the FOX family were studied in 525 primary ccRCC tumors from patients with detailed FOX family gene expression data, and related clinical follow-up data was selected from the updated TCGA data portal. Patients included a total of 184 females and 341 males (age range, 26-90 years; median age, 61 years). All patients had received partial or radical nephrectomy. The enrolled patients had not received pretreatment and had fully characterized tumors, complete RNA sequencing information and intact overall survival (OS) and disease-free survival (DFS) information. Appropriate genes were selected to construct gene networks according to the standards described in a previous study (10). Furthermore, clinicopathological characteristics, including sex, age, tumor diameter, laterality, tumor-node-metastasis, tumor grade, American Joint Committee on Cancer (AJCC) stage (11), levels of white blood cells, platelets and hemoglobin, OS and DFS, were also collected. A network of prognostic FOX genes was obtained from the cBioPortal (http://www.cbioportal.org), and the following criteria were used to construct the network: 'In the same complex', 'interacted with each other' and 'more than $12 \%$ changes'. Unigene accession numbers were obtained from https://www.ncbi.nlm.nih.gov/unigene.

Statistical analysis. Duration of DFS was calculated from the date of diagnosis to the date of first recurrence or mortality. Duration of OS was calculated from the date of diagnosis to the date of mortality or last follow-up which undertaken for a median of 35.95 months. Patients without recurrence or did not succumb to disease were marked as censored at the time of the last follow-up. The Kaplan-Meier method was used for survival analysis, and the log-rank test was used for comparing cumulative survival. The association between overall survival and FOX gene expression was analyzed by performing univariate and multivariate analysis using Cox proportional-hazards regression. All the statistical tests were performed using SPSS (version 22.0; IBM SPSS, Armonk, NY, USA). P<0.05 was considered to indicate a statistically significant difference.

\section{Results}

Clinical characteristics of patients with ccRCC in the TCGA cohort. A total of 525 patients were enrolled in the present study. The patients included 184 females and 341 males with a range of 26-90 years and a median of 61 years. Among the 525 patients, $45.7 \%$ of the patients had low-grade (grade 1 and 2) ccRCC, $52.8 \%$ had high-grade ccRCC and only 8 cases were of undetermined grade. The clinicopathological characteristics
Table I. Clinical characteristics of 525 patients with clear cell renal cell carcinoma in The Cancer Genome Atlas cohort.

\begin{tabular}{|c|c|}
\hline Variables & Patients \\
\hline Age, median (range) & $61(26.0-90.0)$ \\
\hline \multicolumn{2}{|l|}{ Sex, n (\%) } \\
\hline Male & $341(65.0)$ \\
\hline Female & $184(35.0)$ \\
\hline \multicolumn{2}{|l|}{ Grade, $\mathrm{n}(\%)$} \\
\hline 1 & $12(2.3)$ \\
\hline 2 & $228(43.4)$ \\
\hline 3 & $202(38.5)$ \\
\hline 4 & $75(14.3)$ \\
\hline Gx & $8(1.5)$ \\
\hline Tumor diameter, mean (range) & $1.67(0.4-4.0)$ \\
\hline \multicolumn{2}{|l|}{$\mathrm{pT}, \mathrm{n}(\%)$} \\
\hline $\mathrm{T} 1$ & $266(50.7)$ \\
\hline $\mathrm{T} 2$ & $68(13.0)$ \\
\hline $\mathrm{T} 3$ & $179(34.1)$ \\
\hline $\mathrm{T} 4$ & $11(2.1)$ \\
\hline \multicolumn{2}{|l|}{$\mathrm{N}, \mathrm{n}(\%)$} \\
\hline No & $237(45.1)$ \\
\hline N1 & $17(3.2)$ \\
\hline $\mathrm{Nx}$ & $271(51.6)$ \\
\hline \multicolumn{2}{|l|}{$\mathrm{M}, \mathrm{n}(\%)$} \\
\hline M0 & $406(77.3)$ \\
\hline M1 & $78(14.9)$ \\
\hline Mx & $25(4.8)$ \\
\hline \multicolumn{2}{|l|}{ Stage $^{\mathrm{a}}, \mathrm{n}(\%)$} \\
\hline I & $262(49.9)$ \\
\hline II & $56(10.7)$ \\
\hline III & $126(24)$ \\
\hline IV & $81(15.4)$ \\
\hline \multicolumn{2}{|l|}{ Laterality, n (\%) } \\
\hline Left & $247(47.0)$ \\
\hline Right & $277(52.8)$ \\
\hline Bilateral & $1(0.2)$ \\
\hline \multicolumn{2}{|l|}{$\mathrm{Hb}, \mathrm{n}(\%)$} \\
\hline Low & $258(49.1)$ \\
\hline Normal & $181(34.5)$ \\
\hline Elevated & $5(1.0)$ \\
\hline Unavailable & $81(15.4)$ \\
\hline \multicolumn{2}{|l|}{ WBC, n (\%) } \\
\hline Low & $45(8.6)$ \\
\hline Normal & $261(49.7)$ \\
\hline Elevated & $162(30.9)$ \\
\hline Unavailable & $94(17.9)$ \\
\hline \multicolumn{2}{|l|}{ PLT, n (\%) } \\
\hline Low & $45(8.6)$ \\
\hline Normal & $352(67.0)$ \\
\hline Elevated & $37(7.0)$ \\
\hline Unavailable & $91(17.3)$ \\
\hline
\end{tabular}

${ }^{a}$ American Joint Committee on Cancer stage. Hb, hemoglobin; WBC, white blood cell; PLT, platelet; pT, pathological T stage; N, node; M, metastasis. 
Table II. Univariate and multivariate Cox proportional hazards analysis of FOX gene expression and overall survival of patients with clear cell renal cell carcinoma in The Cancer Genome Atlas cohort.

\begin{tabular}{|c|c|c|c|c|}
\hline \multirow[b]{2}{*}{ Variables } & \multicolumn{2}{|c|}{ Univariate } & \multicolumn{2}{|c|}{ Multivariate } \\
\hline & HR $(95 \% \mathrm{CI})$ & P-value & HR $(95 \%$ CI) & P-value \\
\hline Age & $1.03(1.01-1.04)$ & $<0.001$ & $1.03(1.02-1.05)$ & $<0.01$ \\
\hline Sex & $0.95(0.69-1.30)$ & 0.75 & $1.00(0.64-1.58)$ & 0.99 \\
\hline Stage $^{a}$ & $1.95(1.71-2.24)$ & $<0.001$ & $1.29(0.65-2.57)$ & 0.47 \\
\hline Grade $^{b}$ & $2.40(1.94-2.97)$ & $<0.001$ & $1.23(0.86-1.75)$ & 0.25 \\
\hline $\mathrm{Hb}$ & $0.56(0.40-0.79)$ & $<0.001$ & $0.79(0.51-1.23)$ & 1.29 \\
\hline WBC & $0.67(0.48-0.92)$ & 0.01 & $0.92(0.59-1.45)$ & 0.73 \\
\hline PLT & $1.71(1.16-2.53)$ & 0.01 & $1.12(0.73-1.71)$ & 0.60 \\
\hline Tumor diameter & $1.22(0.98-1.50)$ & 0.07 & $0.72(0.52-0.99)$ & 0.04 \\
\hline Position $^{c}$ & $0.70(0.51-0.94)$ & 0.02 & $0.85(0.56-1.27)$ & 0.42 \\
\hline \multicolumn{5}{|l|}{ TNM stage } \\
\hline Tumor & $2.00(1.69-2.36)$ & $<0.001$ & $1.12(0.58-2.16)$ & 0.75 \\
\hline Node & $1.00(0.56-1.75)$ & 0.98 & $0.57(0.28-1.19)$ & 0.13 \\
\hline Metastasis & $4.55(3.31-6.26)$ & $<0.001$ & $3.13(1.15-8.48)$ & 0.03 \\
\hline \multicolumn{5}{|c|}{ FOX family of genes } \\
\hline FOXL2 & $1.25(1.08-1.45)$ & $<0.001$ & $1.27(0.95-1.70)$ & 0.11 \\
\hline FOXL1 & $1.22(1.07-1.39)$ & $<0.001$ & $1.26(1.01-1.57)$ & 0.04 \\
\hline FOXS1 & $1.16(1.03-1.30)$ & 0.01 & $0.93(0.71-1.21)$ & 0.57 \\
\hline FOXN1 & $1.20(0.99-1.45)$ & 0.06 & & \\
\hline FOXN2 & $0.78(0.61-0.99)$ & 0.03 & $0.62(0.39-0.98)$ & 0.04 \\
\hline FOXN3 & $0.59(0.47-0.74)$ & $<0.001$ & $1.07(0.63-1.80)$ & 0.81 \\
\hline FOXHI & $1.29(1.16-1.44)$ & $<0.001$ & $0.85(0.68-1.05)$ & 0.13 \\
\hline FOXG1 & $1.22(1.11-1.34)$ & $<0.001$ & $1.00(0.84-1.18)$ & 0.96 \\
\hline FOXP2 & $1.01(0.94-1.07)$ & 0.88 & & \\
\hline FOXD1 & $1.26(1.14-1.39)$ & $<0.001$ & $0.83(0.70-0.99)$ & 0.04 \\
\hline FOXC2 & $0.95(0.86-1.05)$ & 0.30 & & \\
\hline FOXCl & $1.10(0.93-1.30)$ & 0.29 & & \\
\hline FOXF1 & $0.94(0.82-1.08)$ & 0.41 & & \\
\hline$F O X F 2$ & $1.13(1.00-1.27)$ & 0.05 & $0.94(0.79-1.14)$ & 0.54 \\
\hline FOXE1 & $1.21(1.12-1.31)$ & $<0.001$ & $1.13(0.99-1.30)$ & 0.08 \\
\hline FОХОЗВ & $0.77(0.59-1.01)$ & 0.06 & & \\
\hline FOXB2 & $0.76(0.51-1.13)$ & 0.18 & & \\
\hline FOXR1 & $1.16(0.60-2.23)$ & 0.66 & & \\
\hline FOXN4 & $1.20(1.06-1.36)$ & $<0.001$ & $0.82(0.61-1.10)$ & 0.18 \\
\hline FOXMI & $1.62(1.43-1.83)$ & $<0.001$ & $0.87(0.66-1.15)$ & 0.32 \\
\hline FOXP3 & $1.25(1.14-1.38)$ & $<0.001$ & $0.99(0.86-1.15)$ & 0.94 \\
\hline FOXP1 & $1.34(1.09-1.64)$ & 0.01 & $1.20(0.78-1.85)$ & 0.41 \\
\hline FOXP4 & $2.02(1.57-2.59)$ & $<0.001$ & $1.49(0.91-2.44)$ & 0.11 \\
\hline FОХОЗ & $0.73(0.56-0.94)$ & 0.01 & $0.64(0.39-1.07)$ & 0.09 \\
\hline FOXO1 & $0.67(0.53-0.85)$ & $<0.001$ & $0.99(0.62-1.57)$ & 0.96 \\
\hline FOXO4 & $0.62(0.46-0.84)$ & $<0.001$ & $0.73(0.40-1.37)$ & 0.33 \\
\hline FOXR2 & $1.31(0.80-2.16)$ & 0.29 & & \\
\hline FOXII & $0.98(0.92-1.03)$ & 0.41 & & \\
\hline FOXI3 & $1.25(0.76-2.07)$ & 0.38 & & \\
\hline FOXI2 & $0.85(0.77-0.93)$ & $<0.001$ & $1.07(0.92-1.25)$ & 0.37 \\
\hline FOXRED2 & $1.12(0.91-1.38)$ & 0.28 & & \\
\hline FOXREDI & $1.23(0.95-1.59)$ & 0.12 & & \\
\hline FOXD4L5 & $0.99(0.61-1.61)$ & 0.98 & & \\
\hline FOXD4L6 & $1.31(1.10-1.57)$ & $<0.001$ & $0.91(0.66-1.26)$ & 0.57 \\
\hline
\end{tabular}


Table II. Continued.

\begin{tabular}{|c|c|c|c|c|}
\hline \multirow[b]{2}{*}{ Variables } & \multicolumn{2}{|c|}{ Univariate } & \multicolumn{2}{|c|}{ Multivariate } \\
\hline & HR $(95 \% \mathrm{CI})$ & P-value & HR $(95 \%$ CI $)$ & P-value \\
\hline FOXD4L1 & $1.35(1.16-1.58)$ & $<0.001$ & $0.79(0.56-1.13)$ & 0.20 \\
\hline FOXD4L2 & $1.26(1.08-1.46)$ & $<0.001$ & $1.40(1.10-1.79)$ & 0.01 \\
\hline FOXD4L3 & $1.37(0.92-2.04)$ & 0.13 & & \\
\hline FOXB1 & $1.18(1.02-1.37)$ & 0.03 & $0.97(0.73-1.28)$ & 0.80 \\
\hline$F O X K 2$ & $2.90(2.02-4.14)$ & $<0.001$ & $2.71(1.29-5.71)$ & 0.01 \\
\hline FOXK1 & $0.98(0.77-1.26)$ & 0.89 & & \\
\hline FOXD3 & $1.29(1.05-1.58)$ & 0.02 & $1.01(0.74-1.39)$ & 0.93 \\
\hline FOXAI & $1.19(1.13-1.27)$ & $<0.001$ & $1.12(1.01-1.24)$ & 0.03 \\
\hline FOXA3 & $1.05(0.96-1.14)$ & 0.29 & & \\
\hline FOXA2 & $1.18(1.10-1.25)$ & $<0.001$ & $1.13(1.02-1.26)$ & 0.02 \\
\hline FOXJ1 & $1.13(1.05-1.21)$ & $<0.001$ & $0.98(0.86-1.112)$ & 0.71 \\
\hline FOXJ2 & $1.02(0.69-1.52)$ & 0.91 & & \\
\hline FOXJ3 & $0.96(0.84-1.11)$ & 0.59 & & \\
\hline FOXE3 & $1.72(1.41-2.09)$ & $<0.001$ & $1.34(0.97-1.86)$ & 0.07 \\
\hline FOXQ1 & $1.01(0.91-1.12)$ & 0.93 & & \\
\hline FOXD4 & $1.16(1.00-1.34)$ & 0.04 & $1.17(0.87-1.57)$ & 0.30 \\
\hline FOXD2 & $1.21(1.01-1.44)$ & 0.04 & $0.87(0.58-1.30)$ & 0.49 \\
\hline
\end{tabular}

${ }^{\mathrm{a} A m e r i c a n}$ Joint Committee on Cancer stage; ${ }^{\mathrm{b}} \mathrm{Fuhrman}$ grade; ${ }^{\mathrm{c}}$ Tumors on the left kidney set as 0 and tumors on the right kidney set as 1 . CI, confidence interval; HR, hazards ratio; FOX, forkhead-box; Hb, preoperative hemoglobin count; WBC, preoperative white blood cell count; PLT, preoperative platelet count; TNM, tumor-node-metastasis.

Table III. Multivariate logistic regression analysis of factors that may affect the expression of FOXA1 and FOXA2 in The Cancer Genome Atlas cohort with clear cell renal cell carcinoma.

\section{A, FOXA1}

\begin{tabular}{lcc}
\hline Variables & OR $(95 \% \mathrm{CI})$ & P-value \\
\hline Age & $1.01(0.99-1.02)$ & 0.57 \\
Sex & $1.02(0.68-1.53)$ & 0.93 \\
Stage $^{\mathrm{b}}$ & $1.32(1.10-1.59)$ & $<0.001^{\mathrm{a}}$ \\
Grade $^{\mathrm{c}}$ & $1.51(1.12-2.04)$ & $0.01^{\mathrm{a}}$ \\
Tumor diameter $_{\text {Position }}$ & $0.96(0.71-1.29)$ & 0.78 \\
\hline
\end{tabular}

\section{$\mathrm{B}$, FOXA2}

\begin{tabular}{lcc}
\hline Variable & OR $(95 \% \mathrm{CI})$ & P-value \\
\hline Age & $1.00(0.98-1.01)$ & 0.68 \\
Sex & $0.66(0.44-0.99)$ & $0.04^{\mathrm{a}}$ \\
Stage $^{\mathrm{b}}$ & $1.25(1.03-1.50)$ & $0.02^{\mathrm{a}}$ \\
Grade $^{\mathrm{c}}$ & $1.18(0.88-1.58)$ & 0.28 \\
Tumor diameter $_{\text {Position }}$ & $1.10(0.82-1.48)$ & 0.54 \\
\hline
\end{tabular}

${ }^{\mathrm{a}} \mathrm{P}<0.05$ was considered statistically significant. ${ }^{\mathrm{b}}$ American Joint Committee on Cancer stage; ${ }^{c}$ Fuhrman grade. CI, confidence interval; OR, odds ratio; FOX, forkhead-box. of the enrolled patients are summarized in Table I. Follow-up was undertaken for a median of 35.95 months. At the end of the follow-up, $31.6 \%$ of patients had succumbed to disease $(166 / 525)$.

Selection of independent prognostic factors for $O S$ in the TCGA cohort among FOX gene family members. The median follow-up duration of the patients was 35.95 months, and 166 patients succumbed to disease during the follow-up period. The results of univariate analysis and multivariate analysis of the potential prognostic factors are shown in Table II. Age, AJCC stage and Fuhrman grade and 37 FOX genes were determined to be potential prognostic factors for OS according to univariate Cox proportional hazards ratio analysis $(\mathrm{P}<0.05$; Table II).

These factors were then analyzed by the multivariate Cox proportional hazards ratio model for analysis of OS (Table II). Following adjustment for all potential prognostic factors, the results indicated that age [odds ratio $(\mathrm{OR})=1.034$, 95\% confidence interval (CI), 1.015-1.052], tumor diameter (OR, 0.718; 95\% CI, 0.523-0.986), metastasis stage (OR, 3.129; 95\% CI, 1.154-8.484), FOXAl (OR, 1.120; 95\% CI, 1.014-1.236), FOXA2 (OR, 1.131; 95\% CI, 1.018-1.256), FOXD1 (OR, 0.829; 95\% CI, 0.695-0.987), FOXD4L2 (OR, 1.404; 95\% CI, 1.104-1.786), FOXK2 (OR, 2.712; 95\% CI, 1.288-5.713), FOXL1 (OR, 1.260; 95\% CI, 1.008-1.574) and FOXN2 (OR, 0.621; 95\% CI, 0.393-0.981) were independent prognostic factors for OS (all $\mathrm{P}<0.05$; Table II).

Kaplan-Meier analysis was performed with the cut-off set at the median expression level of each FOX family gene. The 

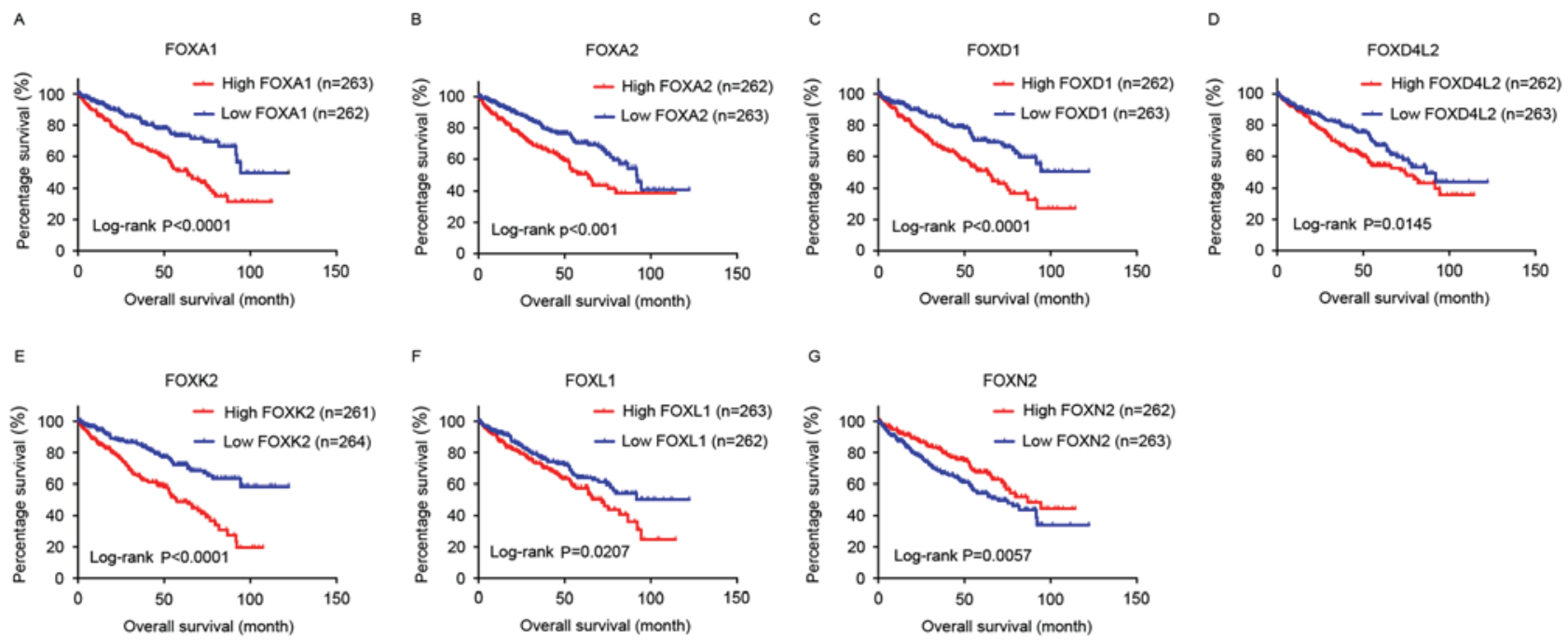

Figure 1. Kaplan-Meier survival curves according to the expression level of FOX family genes and OS in the The Cancer Genome Atlas cohort. (A-G) Kaplan-Meier estimates of OS according to the expression level of (A) FOXA1, (B) FOXA2, (C) FOXD1, (D) FOXD4L2, (E) FOXK2, (F) FOXL1 and (G) FOXN2, respectively. FOX, forkhead-box. OS, overall survival.
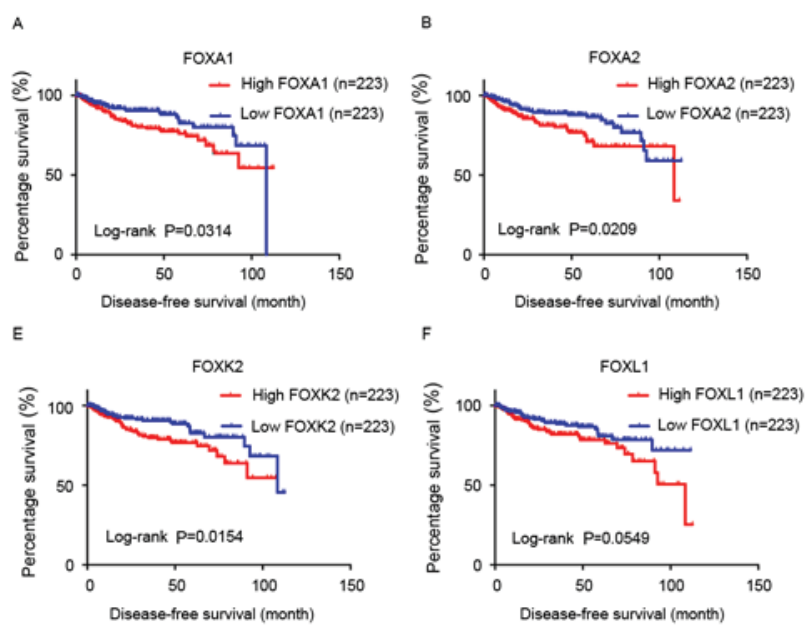
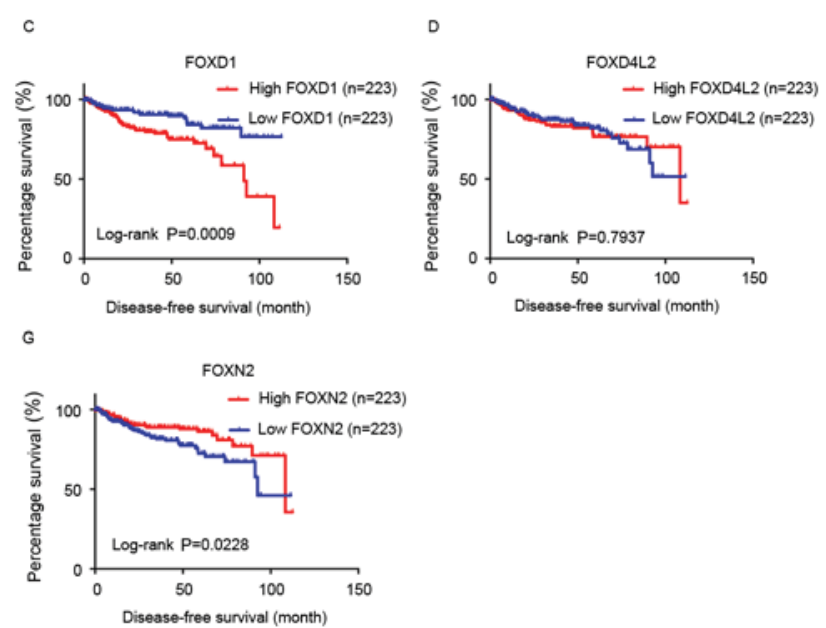

Figure 2. Kaplan-Meier survival curves according to the expression level of FOX family genes and DFS in The Cancer Genome Atlas cohort. Kaplan-Meier estimates of DFS according to the expression level of (A) FOXA1, (B) FOXA2, (C) FOXD1, (D) FOXD4L2, (E) FOXK2, (F) FOXL1 and (G) FOXN2, respectively. FOX, forkhead-box. DFS, disease-free survival.

results revealed that low levels of $F O X A 1, F O X A 2, F O X D 1$ and FOXK2 were associated with longer OS and DFS $(\mathrm{P}<0.05)$, while a high level of FOXN2 was associated with longer OS and DFS (P<0.05; Figs. 1 and 2). A low level of FOXL1 and FOXD4L2 was only associated with longer OS and not DFS $(\mathrm{P}<0.05$; Figs. 1 and 2$)$.

To investigate the association between FOX gene expression and clinical factors, multivariate logistic regression analysis was performed. The results indicated that FOXA1 expression was significantly associated with tumor stage $(\mathrm{P}<0.001$, OR, 1.32; 95\% CI, 1.10-1.59) and grade $(\mathrm{P}=0.01, \mathrm{OR}$, 1.51; 95\% CI, 1.12-2.04) (Table III). FOXA2 was associated with gender $(\mathrm{P}=0.04, \mathrm{OR}, 0.66$; 95\% CI, 0.44-0.99) and stage ( $\mathrm{P}=0.02$, OR, 1.25, 95\% CI, 1.03-1.50) (Table III). FOXDI was only associated with grade $(\mathrm{P}<0.001, \mathrm{OR}, 1.65 ; 95 \% \mathrm{CI}$, 1.22-2.23). FOXK2 was associated with gender $(\mathrm{P}<0.001$, OR, 0.54; 95\% CI, 0.36-0.81) and tumor grade $(\mathrm{P}=0.02$, OR, 1.44; 95\% CI, 1.07-1.94). FOXL1 was associated with gender
$(\mathrm{P}=0.04$, OR, 1.53; 95\% CI, 1.03-2.26). However, no significant association was observed between FOXD4L2, FOXN2 and clinical variables (Tables IV and V).

FOX gene network revealed nuclear receptor coactivator (NCOA)1, NADH dehydrogenase (ubiquinone) flavoprotein (NDUFV)3, phosphatidylserine decarboxylase (PISD) and pyruvate kinase, liver and red blood cell (PKLR) are independent prognostic factors for OS in the TCGA cohort. It was investigated whether the expression level of FOX family-associated genes had an effect on patient OS in TCGA cohort. The gene network is shown in Fig. 3, and details of the genes in the network are shown in Table VI. The data from univariate Cox proportional hazards ratio analysis indicated that the expression levels of acyl-coenzyme A dehydrogenase, C-4 to C-12 straight chain, androgen receptor, $\alpha$-fetoprotein, bone morphogenetic protein receptor type II, CCAAT/enhancer-binding protein $\beta$, engrailed homeobox 2 , hydroxyacyl-coenzyme A 
Table IV. Multivariate logistic regression analysis of factors that may affect the expression of FOXD1 and FOXD4L1 in The Cancer Genome Atlas cohort with clear cell renal cell carcinoma.

\section{A, FOXD1}

\begin{tabular}{lcc}
\hline Variable & OR $(95 \% \mathrm{CI})$ & P-value \\
\hline Age & $1.01(0.99-1.02)$ & 0.32 \\
Sex & $1.43(0.96-2.15)$ & 0.08 \\
Stage $^{\mathrm{b}}$ & $1.12(0.93-1.34)$ & 0.25 \\
Grade $^{\mathrm{c}}$ & $1.65(1.22-2.23)$ & $<0.001^{\mathrm{a}}$ \\
Tumor diameter $^{\mathrm{A}}$ & $1.02(0.76-1.38)$ & 0.89 \\
Position & $1.15(0.79-1.67)$ & 0.48 \\
\hline
\end{tabular}

\section{B, FOXD4L1}

\begin{tabular}{lcc}
\hline Variable & OR $(95 \% \mathrm{CI})$ & P-value \\
\hline Age & $1.01(0.99-1.02)$ & 0.37 \\
Sex & $0.75(0.50-1.11)$ & 0.15 \\
Stage $^{\mathrm{b}}$ & $1.20(1.00-1.44)$ & 0.05 \\
Grade $^{\mathrm{c}}$ & $1.11(0.83-1.49)$ & 0.48 \\
Tumor diameter $_{\text {Position }}$ & $1.18(0.88-1.58)$ & 0.28 \\
& $0.72(0.49-1.04)$ & 0.08
\end{tabular}

${ }^{\mathrm{a}} \mathrm{P}<0.05$ was considered statistically significant; ${ }^{\mathrm{b}}$ American Joint Committee on Cancer stage; ${ }^{\mathrm{c}}$ Fuhrman grade. CI, confidence interval; OR, odds ratio; FOX, forkhead-box.

dehydrogenase, 3-hydroxy-3-methylglutaryl-coenzyme A synthase 1 , hepatocyte nuclear factor $4 \alpha$, insulin-like growth factor binding protein 1 , interleukin-2 $(I L-2)$, potassium inwardly-rectifying channel subfamily $\mathrm{J}$ member 11, NCOA1, NCOA3, NDUFV3, PISD, PKLR and uncoupling protein 2 were associated with OS. Multivariate analysis for prognostic factors was performed by the Cox proportional hazards ratio analysis and revealed that the expression of NCOA1, NDUFV3, $P I S D$ and $P K L R$ were independent prognostic factors for OS in the TCGA cohort (Table VII).

\section{Discussion}

In the present study, it was revealed that FOXA1, FOXA2, FOXD1, FOXD4L2, FOXK2 and FOXL1 genes were risk factors for clinical outcome of ccRCC. However, high expression of the FOXN2 gene was associated with longer survival in the TCGA cohort. Furthermore, in a network of FOX family-related genes, NCOA1, NDUFV3, PISD and PKLR were identified as independent prognostic factors for OS in patients with ccRCC.

FOXA1 and FOXA2 are two members of the FOXA transcription factor family. FOXA1, also termed HNF-3, has an important role in the progression of bladder, prostate and breast cancer (12-15). A previous study has demonstrated that downregulation of FOXA1 is associated with poor OS in human bladder cancer (12). FOXA1 may also be a
Table V. Multivariate logistic regression analysis of factors that may affect the expression of FOXK2, FOXL1 and FOXN2 in The Cancer Genome Atlas cohort with clear cell renal cell carcinoma.

\section{A, FOXK2}

\begin{tabular}{lcc}
\hline Variable & OR $(95 \% \mathrm{CI})$ & P-value \\
\hline Age & $1.00(0.98-1.01)$ & 0.56 \\
Sex & $0.54(0.36-0.81)$ & $<0.001^{\mathrm{a}}$ \\
Stage $^{\mathrm{b}}$ & $1.10(0.92-1.33)$ & 0.30 \\
Grade $^{\mathrm{c}}$ & $1.44(1.07-1.94)$ & $0.02^{\mathrm{a}}$ \\
Tumor diameter $_{\text {Position }}$ & $1.03(0.77-1.39)$ & 0.82 \\
\hline
\end{tabular}

\section{B, FOXL1}

\begin{tabular}{lcc}
\hline Variable & OR $(95 \% \mathrm{CI})$ & P-value \\
\hline Age & $1.00(0.99-1.02)$ & 0.97 \\
Sex & $1.53(1.03-2.26)$ & $0.04^{\mathrm{a}}$ \\
Stage $^{\mathrm{b}}$ & $1.13(0.94-1.35)$ & 0.20 \\
Grade $^{\mathrm{c}}$ & $1.00(0.75-1.34)$ & 0.99 \\
Tumor diameter $_{\text {Position }}$ & $0.87(0.65-1.16)$ & 0.34 \\
\hline
\end{tabular}

\section{C, FOXN2}

\begin{tabular}{lcc}
\hline Variable & OR $(95 \% \mathrm{CI})$ & P-value \\
\hline Age & $0.99(0.97-1.00)$ & 0.13 \\
Sex & $1.20(0.80-1.79)$ & 0.38 \\
Stage $^{\mathrm{b}}$ & $0.85(0.71-1.03)$ & 0.09 \\
Grade $^{\mathrm{c}}$ & $0.89(0.67-1.20)$ & 0.46 \\
Tumor diameter $_{\text {Position }}$ & $0.79(0.59-1.06)$ & 0.12 \\
\hline
\end{tabular}

${ }^{\mathrm{a}} \mathrm{P}<0.05$ was considered statistically significant; ${ }^{\mathrm{b}}$ American Joint Committee on Cancer stage; ${ }^{~}$ Fuhrman grade. CI, confidence interval; OR, odds ratio; FOX, forkhead-box.

potential treatment target of breast and prostate cancer due to its effects on chromatin remodeling via androgen and estrogen receptors (16). FOXA2 is involved in proliferation, differentiation and maintenance of cancer stem cells (17-19). However, FOXA2 may have different roles in different tissues. FOXA2 is associated with the prognosis of human gastric cancer, and patients with high FOXA2 expression level had longer OS compared with patients with low FOXA2 expression (19). However, one study conducted in breast carcinoma revealed that FOXA2 promotes the development of triple-negative/basal-like tumors (18).

FOXD1 performs an essential role in numerous biological processes, including proliferation, differentiation and tumorigenesis $(20,21)$. Upregulation of FOXD1 is associated with the development of resistance to chemotherapy 
Table VI. List of FOX family-associated genes as revealed by gene network analysis.

\begin{tabular}{|c|c|c|}
\hline Gene & Full gene name & UniGene $^{\mathrm{a}}$ \\
\hline$F O X K 2$ & Forkhead box K2 & Hs.591140 \\
\hline$X B P 1$ & X-box binding protein 1 & Hs.437638 \\
\hline FOXL1 & Forkhead box L1 & Hs.533830 \\
\hline EN2 & Engrailed homeobox 2 & Hs.134989 \\
\hline FOXA2 & Forkhead box A2 & Hs.155651 \\
\hline$F O X F 1$ & Forkhead box F1 & Hs.155591 \\
\hline$C E B P B$ & CCAAT/enhancer-binding protein $\beta$ & Hs.517106, Hs.716248 \\
\hline FOXAl & Forkhead box A1 & Hs.163484 \\
\hline$H A D H$ & Hydroxyacyl-coenzyme A dehydrogenase & Hs.438289 \\
\hline$B D H 1$ & 3-hydroxybutyrate dehydrogenase, type 1 & Hs.274539 \\
\hline$A C A D M$ & Acyl-coenzyme A dehydrogenase, $\mathrm{C}-4$ to $\mathrm{C}-12$ straight chain & Hs.445040 \\
\hline$A C A D V L$ & Acyl-coenzyme A dehydrogenase, very long chain & Hs.463928, Hs.437178 \\
\hline$A P 1 B 1$ & Adaptor-related protein complex $1, \beta 1$ subunit & Hs.368794 \\
\hline HMGCS1 & 3-hydroxy-3-methylglutaryl-coenzyme A synthase 1 (soluble) & Hs.397729 \\
\hline$B M P R 2$ & Bone morphogenetic protein receptor type II (serine/threonine kinase) & Hs.471119 \\
\hline$N R 3 C 1$ & Nuclear receptor subfamily 3 , group C, member 1 (glucocorticoid receptor) & Hs.122926 \\
\hline KCNJ11 & Potassium inwardly-rectifying channel subfamily J member 11 & Hs.248141 \\
\hline $\mathrm{SHH}$ & Sonic hedgehog homolog (Drosophila) & Hs.164537 \\
\hline$A K T 1$ & V-Akt murine thymoma viral oncogene homolog 1 & Hs.525622 \\
\hline$A P O B$ & Apolipoprotein B (including $\mathrm{Ag}(\mathrm{x})$ antigen) & Hs.120759 \\
\hline HOXA5 & Homeobox A5 & Hs.655218 \\
\hline$S L C 2 A 2$ & Solute carrier family 2 (facilitated glucose transporter), member 2 & Hs.167584 \\
\hline SERPINAI & Serpin peptidase inhibitor, clade A ( $\alpha-1$ antiproteinase, antitrypsin), member 1 & Hs.525557 \\
\hline$N R 2 F 2$ & Nuclear receptor subfamily 2 , group F, member 2 & Hs.657455, Hs.347991 \\
\hline DSCAM & Down syndrome cell adhesion molecule & Hs.397800 \\
\hline COL18A1 & Collagen, type XVIII, $\alpha 1$ & Hs.517356 \\
\hline$A R$ & Androgen receptor & Hs.496240 \\
\hline$K L K 3$ & Kallikrein-related peptidase 3 & Hs.171995 \\
\hline OTX2 & Orthodenticle homeobox 2 & Hs.288655 \\
\hline$P I S D$ & Phosphatidylserine decarboxylase & Hs.420559 \\
\hline SOD1 & Superoxide dismutase 1 , soluble & Hs.443914 \\
\hline NRIP1 & Nuclear receptor interacting protein 1 & Hs.155017 \\
\hline NDUFV3 & NADH dehydrogenase (ubiquinone) flavoprotein 3, $10 \mathrm{kDa}$ & Hs.473937 \\
\hline$A F P$ & $\alpha$-fetoprotein & Hs.518808 \\
\hline NCOAl & Nuclear receptor coactivator 1 & Hs.596314 \\
\hline$C D K N 1 B$ & Cyclin-dependent kinase inhibitor 1B (p27, Kip1) & Hs.238990 \\
\hline NCOA3 & Nuclear receptor coactivator 3 & Hs.592142 \\
\hline$H N F 4 A$ & Hepatocyte nuclear factor $4 \alpha$ & Hs.116462 \\
\hline$U C P 2$ & Uncoupling protein 2 (mitochondrial, proton carrier) & Hs.80658 \\
\hline$P K L R$ & Pyruvate kinase, liver and red blood cell & Hs.95990 \\
\hline$I L-2$ & Interleukin 2 & Hs.89679 \\
\hline
\end{tabular}

${ }^{a}$ Accession numbers (https://www.ncbi.nlm.nih.gov/unigene). Hs, Homo sapiens.

in patients with prostate and ovarian cancer (22). Another study reported that FOXD1 is upregulated in breast cancer, and the depletion or overexpression of FOXD1 may cause changes in proliferation and chemoresistance (20). FOXK2, also termed ILF or ILF1, was first identified as a regulator of IL-2 transcription. FOXK2 upregulates activator protein-1 (AP-1)-dependent gene expression through its interaction with AP-1 and accelerates the binding of AP-1 to chromatin (23). FOXL1 is associated with pancreatic carcinoma and has an important inhibitory role in pancreatic tumor progression (24). However, to the best of our knowledge, no study has examined FOXD4L2 to date, and the findings of the present study suggest that FOXD4L2 should be investigated further in future studies. In addition, it was observed 
Table VII. Cox proportional hazards analysis of FOX family genes, related gene network, clinical parameters and overall survival for The Cancer Genome Atlas clear cell renal cell carcinoma cohort.

\begin{tabular}{|c|c|c|c|c|}
\hline \multirow[b]{2}{*}{ Variables } & \multicolumn{2}{|c|}{ Univariate } & \multicolumn{2}{|c|}{ Multivariate } \\
\hline & HR $(95 \% \mathrm{CI})$ & P-value & HR $(95 \%$ CI $)$ & P-value \\
\hline \multicolumn{5}{|l|}{ Demographic parameters } \\
\hline Age & $1.028(1.015-1.041)$ & $<0.0001$ & $1.046(1.024-1.068)$ & $<0.0001$ \\
\hline Sex (male vs. female) & $0.950(0.693-1.302)$ & 0.752 & $0.722(0.390-1.336)$ & 0.300 \\
\hline \multicolumn{5}{|l|}{ Clinical parameters } \\
\hline Stage $^{\mathrm{a}}(\mathrm{I}-\mathrm{IV})$ & $1.954(1.707-2.236)$ & $<0.0001$ & & \\
\hline $\operatorname{Grade}^{\mathrm{b}}(\mathrm{I}-\mathrm{IV})$ & $2.399(1.941-2.965)$ & $<0.0001$ & $1.717(1.104-2.672)$ & 0.016 \\
\hline Tumor diameter & $1.215(0.983-1.502)$ & 0.071 & $0.600(0.412-0.873)$ & 0.008 \\
\hline Laterality (left vs. right) & $0.695(0.512-0.944)$ & 0.020 & $0.665(0.412-1.074)$ & 0.096 \\
\hline pT (T1/T2/T3) & $1.992(1.685-2.355)$ & $<0.0001$ & $1.337(0.954-1.874)$ & 0.092 \\
\hline pN (N1 vs. N2) & $0.992(0.562-1.752)$ & 0.978 & $0.552(0.236-1.292)$ & 0.171 \\
\hline pM (M0 vs. M1) & $4.548(3.305-6.257)$ & $<0.0001$ & $6.362(3.172-12.757)$ & $<0.0001$ \\
\hline $\mathrm{Hb}$ (low/normal/elevated) & $0.563(0.400-0.792)$ & 0.001 & $0.665(0.391-1.131)$ & 0.132 \\
\hline WBC (low/normal/elevated) & $0.668(0.483-0.923)$ & 0.014 & $0.766(0.434-1.354)$ & 0.360 \\
\hline PLT (low/normal/elevated) & $1.709(1.156-2.526)$ & 0.007 & $1.443(0.912-2.285)$ & 0.117 \\
\hline \multicolumn{5}{|l|}{ FOX family genes } \\
\hline FOXL2 & $1.250(1.076-1.452)$ & 0.004 & $1.882(1.283-2.760)$ & 0.001 \\
\hline FOXL1 & $1.219(1.071-1.387)$ & 0.003 & $1.418(1.056-1.904)$ & 0.020 \\
\hline FOXSI & $1.160(1.031-1.303)$ & 0.013 & $0.648(0.436-0.964)$ & 0.032 \\
\hline FOXN1 & $1.200(0.992-1.451)$ & 0.060 & & \\
\hline FOXN2 & $0.775(0.614-0.978)$ & 0.032 & $0.253(0.103-0.618)$ & 0.003 \\
\hline FOXN3 & $0.589(0.470-0.740)$ & $<0.0001$ & $0.515(0.228-1.163)$ & 0.110 \\
\hline FOXHI & $1.292(1.156-1.444)$ & $<0.0001$ & $0.825(0.603-1.130)$ & 0.231 \\
\hline FOXG1 & $1.217(1.110-1.335)$ & $<0.0001$ & $0.825(0.658-1.034)$ & 0.095 \\
\hline FOXP2 & $1.005(0.942-1.072)$ & 0.875 & & \\
\hline FOXD1 & $1.261(1.142-1.392)$ & $<0.0001$ & $0.703(0.554-0.891)$ & 0.004 \\
\hline FOXC2 & $0.948(0.855-1.050)$ & 0.304 & & \\
\hline FOXC1 & $1.095(0.926-1.296)$ & 0.288 & & \\
\hline FOXF1 & $0.942(0.819-1.084)$ & 0.406 & & \\
\hline FOXF2 & $1.129(1.000-1.274)$ & 0.050 & $0.942(0.741-1.197)$ & 0.625 \\
\hline FOXE1 & $1.212(1.120-1.312)$ & $<0.0001$ & $1.175(0.983-1.405)$ & 0.077 \\
\hline FОХОЗВ & $0.774(0.591-1.012)$ & 0.061 & & \\
\hline$F O X B 2$ & $0.762(0.513-1.130)$ & 0.177 & & \\
\hline FOXRl & $1.160(0.604-2.228)$ & 0.655 & & \\
\hline FOXN4 & $1.200(1.062-1.357)$ & 0.003 & $0.615(0.421-0.899)$ & 0.012 \\
\hline FOXMI & $1.618(1.433-1.827)$ & $<0.0001$ & $0.950(0.638-1.414)$ & 0.800 \\
\hline FOXP3 & $1.252(1.141-1.375)$ & $<0.0001$ & $0.926(0.713-1.203)$ & 0.565 \\
\hline FOXP1 & $1.336(1.089-1.639)$ & 0.006 & $1.761(0.820-3.781)$ & 0.147 \\
\hline FOXP4 & $2.018(1.572-2.591)$ & 0.000 & $1.695(0.900-3.191)$ & 0.102 \\
\hline FOXO3 & $0.726(0.562-0.938)$ & 0.014 & $0.393(0.199-0.779)$ & 0.007 \\
\hline FOXO1 & $0.671(0.529-0.851)$ & 0.001 & $1.978(0.973-4.021)$ & 0.059 \\
\hline FOXO4 & $0.624(0.464-0.840)$ & 0.002 & $0.521(0.228-1.194)$ & 0.123 \\
\hline FOXR2 & $1.310(0.795-2.160)$ & 0.290 & & \\
\hline FOXII & $0.976(0.920-1.034)$ & 0.409 & & \\
\hline FOXI3 & $1.253(0.757-2.074)$ & 0.379 & & \\
\hline FOXI2 & $0.848(0.772-0.930)$ & 0.001 & $0.999(0.816-1.222)$ & 0.990 \\
\hline FOXRED2 & $1.121(0.909-1.383)$ & 0.284 & & \\
\hline FOXREDI & $1.231(0.950-1.594)$ & 0.115 & & \\
\hline FOXD4L5 & $0.994(0.612-1.614)$ & 0.980 & & \\
\hline FOXD4L6 & $1.312(1.100-1.566)$ & 0.003 & $0.909(0.607-1.362)$ & 0.644 \\
\hline
\end{tabular}


Table VII. Continued.

\begin{tabular}{|c|c|c|c|c|}
\hline \multirow[b]{2}{*}{ Variables } & \multicolumn{2}{|c|}{ Univariate } & \multicolumn{2}{|c|}{ Multivariate } \\
\hline & HR $(95 \% \mathrm{CI})$ & P-value & HR $(95 \% \mathrm{CI})$ & P-value \\
\hline FOXD4L1 & $1.352(1.158-1.578)$ & $<0.0001$ & $0.841(0.546-1.296)$ & 0.433 \\
\hline$F O X D 4 L 2$ & $1.256(1.082-1.458)$ & 0.003 & $1.450(1.059-1.986)$ & 0.021 \\
\hline FOXD4L3 & $1.366(0.91-2.036)$ & 0.126 & & \\
\hline FOXB1 & $1.183(1.017-1.375)$ & 0.029 & $0.954(0.652-1.394)$ & 0.806 \\
\hline FOXK2 & $2.895(2.023-4.142)$ & $<0.0001$ & $1.164(0.356-3.798)$ & 0.802 \\
\hline FOXK1 & $0.982(0.765-1.260)$ & 0.885 & & \\
\hline FOXD3 & $1.286(1.049-1.577)$ & 0.016 & $0.930(0.637-1.358)$ & 0.708 \\
\hline FOXAI & $1.194(1.127-1.266)$ & $<0.0001$ & $1.224(1.066-1.405)$ & 0.004 \\
\hline FOXA3 & $1.047(0.962-1.140)$ & 0.290 & & \\
\hline FOXA2 & $1.175(1.103-1.253)$ & $<0.0001$ & $1.153(1.010-1.316)$ & 0.035 \\
\hline FOXJI & $1.126(1.046-1.213)$ & 0.002 & $1.074(0.914-1.316)$ & 0.383 \\
\hline FOXJ2 & $1.024(0.688-1.524)$ & 0.907 & & \\
\hline FOXJ3 & $0.963(0.838-1.106)$ & 0.591 & & \\
\hline FOXE3 & $1.715(1.409-2.088)$ & $<0.0001$ & $1.374(0.923-2.045)$ & 0.118 \\
\hline$F O X Q 1$ & $1.005(0.905-1.115)$ & 0.929 & & \\
\hline FOXD4 & $1.159(1.004-1.337)$ & 0.044 & $1.063(0.735-1.538)$ & 0.744 \\
\hline FOXD2 & $1.206(1.011-1.440)$ & 0.038 & $0.960(0.543-1.697)$ & 0.889 \\
\hline \multicolumn{5}{|c|}{ Network genes } \\
\hline$B D H 1$ & $1.047(0.955-1.148)$ & 0.331 & $0.945(0.777-1.149)$ & 0.570 \\
\hline EN2 & $1.258(1.173-1.350)$ & $<0.0001$ & $0.930(0.800-1.080)$ & 0.341 \\
\hline$P K L R$ & $0.898(0.852-0.947)$ & $<0.0001$ & $0.794(0.659-0.955)$ & 0.015 \\
\hline$A C A D V L$ & $1.211(0.949-1.546)$ & 0.123 & $0.774(0.388-1.546)$ & 0.468 \\
\hline$U C P 2$ & $1.292(1.108-1.508)$ & 0.001 & $1.217(0.791-1.871)$ & 0.372 \\
\hline KCNJI1 & $1.108(1.000-1.226)$ & 0.049 & $0.936(0.698-1.256)$ & 0.661 \\
\hline HOXA5 & $0.985(0.842-1.153)$ & 0.854 & $1.748(1.214-2.518)$ & 0.003 \\
\hline OTX2 & $1.263(0.970-1.646)$ & 0.083 & $0.740(0.466-1.176)$ & 0.202 \\
\hline$N R 3 C 1$ & $0.823(0.642-1.054)$ & 0.123 & $0.861(0.421-1.763)$ & 0.683 \\
\hline$H N F 4 A$ & $0.916(0.866-0.969)$ & 0.002 & $1.011(0.831-1.229)$ & 0.916 \\
\hline IL2 & $1.339(1.100-1.631)$ & 0.004 & $0.874(0.589-1.298)$ & 0.506 \\
\hline$C D K N 1 B$ & $0.750(0.557-1.010)$ & 0.058 & $2.720(1.215-6.093)$ & 0.015 \\
\hline$A R$ & $0.798(0.744-0.857)$ & $<0.0001$ & $1.023(0.795-1.317)$ & 0.860 \\
\hline NCOAl & $0.523(0.385-0.711)$ & $<0.0001$ & $3.901(1.399-10.875)$ & 0.009 \\
\hline COL18A1 & $1.143(0.933-1.400)$ & 0.196 & $1.024(0.611-1.715)$ & 0.929 \\
\hline$H M G C S 1$ & $0.524(0.386-0.711)$ & $<0.0001$ & $0.962(0.519-1.783)$ & 0.903 \\
\hline$A C A D M$ & $0.537(0.454-0.634)$ & $<0.0001$ & $1.011(0.561-1.822)$ & 0.971 \\
\hline$S L C 2 A 2$ & $0.926(0.879-0.976)$ & 0.004 & $1.045(0.882-1.237)$ & 0.614 \\
\hline$A K T 1$ & $1.442(0.943-2.204)$ & 0.091 & $1.924(0.612-6.044)$ & 0.263 \\
\hline$B M P R 2$ & $0.608(0.475-0.777)$ & $<0.0001$ & $0.929(0.369-2.337)$ & 0.875 \\
\hline$X B P 1$ & $1.032(0.826-1.289)$ & 0.783 & $0.647(0.410-1.023)$ & 0.062 \\
\hline DSCAM & $1.076(0.971-1.191)$ & 0.162 & $1.262(1.047-1.520)$ & 0.014 \\
\hline NDUFV3 & $1.560(1.144-2.126)$ & 0.005 & $2.021(1.080-3.785)$ & 0.028 \\
\hline SOD1 & $1.280(0.954-1.718)$ & 0.100 & $1.191(0.494-2.871)$ & 0.697 \\
\hline$C E B P B$ & $1.525(1.336-1.741)$ & $<0.0001$ & $0.905(0.627-1.305)$ & 0.592 \\
\hline NCOA3 & $0.701(0.534-0.920)$ & 0.011 & $1.128(0.407-3.126)$ & 0.816 \\
\hline$A P 1 B 1$ & $1.023(0.728-1.436)$ & 0.897 & $0.502(0.203-1.305)$ & 0.134 \\
\hline$K L K 3$ & $0.990(0.892-1.099)$ & 0.853 & $0.895(0.630-1.272)$ & 0.537 \\
\hline $\mathrm{SHH}$ & $0.990(0.893-1.098)$ & 0.855 & $0.930(0.765-1.130)$ & 0.463 \\
\hline PISD & $2.048(1.617-2.595)$ & $<0.0001$ & $3.389(1.722-6.667)$ & $<0.0001$ \\
\hline$A F P$ & $1.124(1.035-1.221)$ & 0.006 & $0.974(0.822-1.155)$ & 0.765 \\
\hline$I G F B P 1$ & $1.095(1.052-1.141)$ & $<0.0001$ & $0.970(0.872-1.080)$ & 0.581 \\
\hline
\end{tabular}


Table VII. Continued.

\begin{tabular}{|c|c|c|c|c|}
\hline \multirow[b]{2}{*}{ Variables } & \multicolumn{2}{|c|}{ Univariate } & \multicolumn{2}{|c|}{ Multivariate } \\
\hline & HR $(95 \% \mathrm{CI})$ & P-value & $\mathrm{HR}(95 \% \mathrm{CI})$ & P-value \\
\hline$A P O B$ & $1.001(0.948-1.057)$ & 0.970 & $1.087(0.962-1.228)$ & 0.183 \\
\hline$N R 2 F 2$ & $0.985(0.765-1.266)$ & 0.903 & $0.558(0.317-0.983)$ & 0.043 \\
\hline SERPINAI & $1.027(0.940-1.122)$ & 0.552 & $1.139(0.899-1.442)$ & 0.281 \\
\hline$H A D H$ & $0.375(0.264-0.533)$ & $<0.0001$ & $0.560(0.235-1.335)$ & 0.191 \\
\hline
\end{tabular}

${ }^{\mathrm{a}}$ American Joint Committee on Cancer stage; ${ }^{\text {} F u h r m a n ~ g r a d e . ~ F O X, ~ f o r k h e a d-b o x ; ~ H b, ~ h e m o g l o b i n ; ~ W B C, ~ w h i t e ~ b l o o d ~ c e l l ; ~ P L T, ~ p l a t e l e t ; ~ p N ~}$ pathological node stage; pM, pathological metastasis stage; pT, pathological T stage; HR, hazard ratio; CI, confidence interval.
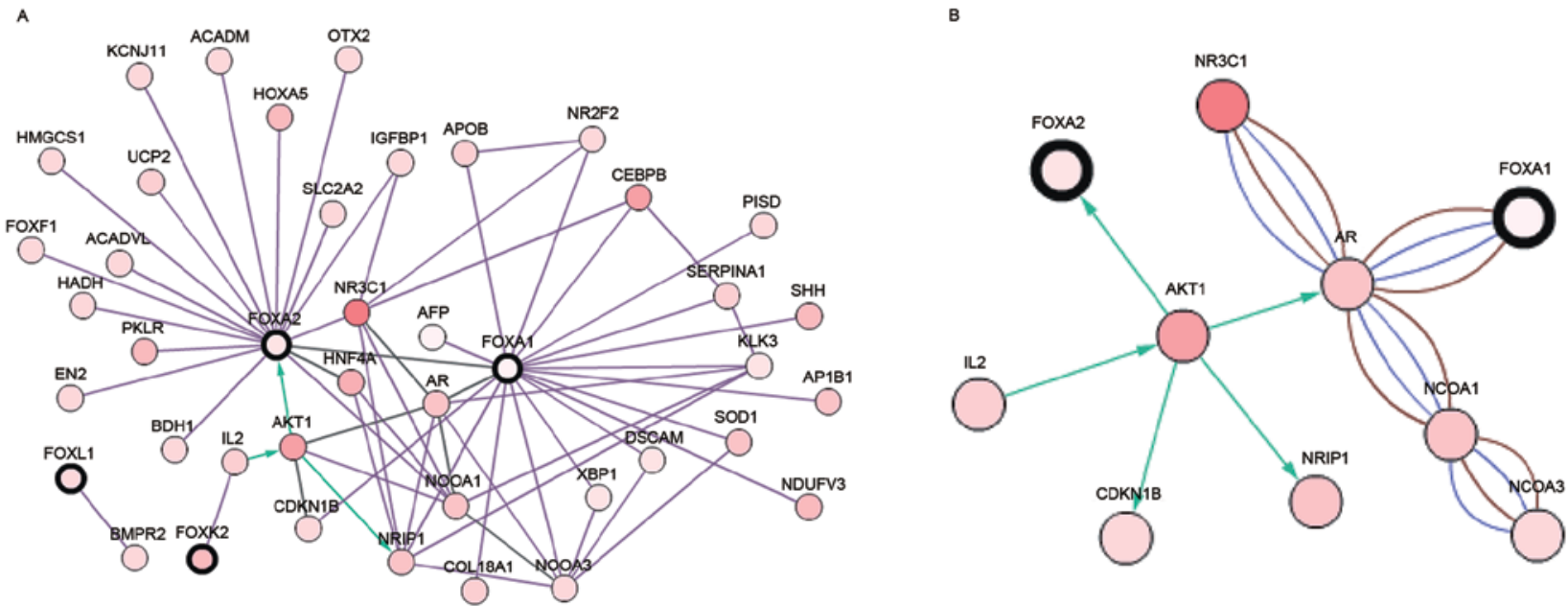

Figure 3. Gene network of prognosis-associated FOX genes. Gene network was drawn with the independent prognosis predictors FOXA1, FOXA2, FOXD1, FOXD4L2, FOXK2, FOXL1 and FOXN2 genes. A total of three criteria were selected for construction of the network: 'Interacts with', 'state change' and 'in the same component'. The threshold of state change was set as $12 \%$. The network was plotted using the cBioPortal website (www.cbioportal.org). (A) FOXN2, FOXD1 and FOXD4L2 were absent in the network since they were not connected with any genes. A network involving FOXA1, FOXA2, FOXK2 and FOXL1 was constructed with the aforementioned conditions. Connecting lines meant an association between the two connected genes. (B) Most significant associations between FOXA1 and FOXA2 were drawn. Brown line, genes in in the same component, blue line, gene interactions; green line, co-expression.

in the present study that high FOXN2 mRNA expression was associated with longer OS, and this is consistent with previous results in glioblastoma multiforme (25).

Previous studies have shown that FOX genes have an essential role in the progression of several types of tumors, including ccRCC $(9,12-14,19,21)$. However, to the best of our knowledge, the present study is the first to comprehensively examine the association between the outcome of ccRCC and the gene expression of the entire FOX gene family. The detailed mechanisms remain unknown and would need to be investigated in future studies.

The present study also investigated the association between FOX-related genes and prognosis of patients with ccRCC. The results indicated that the FOX-associated genes NCOA1, NDUFV3, PISD and PKLR are associated with OS of patients with ccRCC. It has been previously demonstrated that the nuclear co-activator NCOA1 (SRC-1) is able to promote breast cancer metastasis through directly targeting macrophage colony-stimulating factor 1 expression (26). Overexpression of NCOA1 is associated with resistance to endocrine therapy and disease recurrence (26). The NDUFV3 gene is located at chromosome 21q22.3 and may be associated with the occurrence of Down syndrome (27). Although limited information is known about PISD, one study reported that PISD was associated with tumorigenesis and tumor growth (28). The PKLR gene is considered to be involved in pyruvate kinase-deficient hemolytic anemia (29).

The major strength of the present study is that it is the first comprehensive evaluation of the association between FOX genes and the prognosis of patients with ccRCC. The study involved a large cohort, and the clinical follow-up was long. These findings will help provide the foundation to elucidate the mechanisms of FOX genes and their function in ccRCC.

However, the present study also has a number of limitations. Firstly, only data from TCGA database was analyzed and further validation is required. Secondly, the present study did not investigate the specific mechanisms of action of FOX genes in patients with ccRCC. Additionally, the FOX gene signature may not be sufficient to predict the prognosis of ccRCC, since other factors (tumor stage, surgical procedures, state of nutrition, economic issues, response to sunitinib, comorbidities and lifestyle factors), can also affect 
the prognosis of ccRCC $(5,9,11,30)$. Therefore, additional study is required to examine the association between FOX genes and ccRCC.

Findings of the present study suggest that the expression of FOX family genes FOXA1, FOXA2, FOXD1, FOXD4L2, FOXK2, FOXL1 and FOXN2 and FOX family-related genes NCOA1, NDUFV3, PISD and PKLR are associated with survival in patients with ccRCC. Findings of the present study and the specific underlying require further investigation.

\section{Acknowledgements}

The authors would like to thank The Cancer Genome Atlas Group and cancer browser website (https://genome-cancer. ucsc.edu/) for the collection of, and the open access to all data.

\section{Funding}

The present study was supported by the International Cooperation and Exchange of Science and Technology Commission of Shanghai Municipality (grant no. 12410709300), the Guide Project of Science and Technology Commission of Shanghai Municipality (grant no. 124119a7300), the Outstanding Young Talent Training Plan of Shanghai Municipal Commission of Health and Family Planning (grant no. XYQ2013102), the National Nature Science Foundation of China (grant nos. 81001131 and 81472377), the Shanghai Municipal Commission of Health and Family Planning grant (grant no. 2014zyjb0102) and the National Science Foundation for Young Scientists of China (grant no. 81202004).

\section{Availability of data and materials}

The datasets generated and analyzed during the current study are available from the Cancer Genomics Browser of University of California Santa Cruz (https://genome-cancer.ucsc.edu/), and the datasets are available on reasonable request from the corresponding author.

\section{Authors' contributions}

ZWJ and FNW conceived the present study, collected and analyzed the clinical data, and drafted the manuscript. BD and DWY designed and supervised this study. YZ and GHS contributed to the collection of the clinical data, and HLZ helped to analyze the data. All authors reviewed and approved the manuscript.

\section{Ethics approval and consent to participate}

The datasets we used in this article were generated and analyzed from The Cancer Genome Atlas Group, which had been approved by Memorial Sloan-Kettering Cancer Center institutional review board (31), and the informed consent was provided at the same time.

\section{Consent for publication}

Not applicable.

\section{Competing interests}

All authors declare that they have no competing interests.

\section{References}

1. Motzer RJ, Agarwal N, Beard C, Bhayani S, Bolger GB, Carducci MA, Chang SS, Choueiri TK, Hancock SL, Hudes GR, et al: Kidney cancer. J Natl Compr Canc Netw 9: 960-977, 2011.

2. Ljungberg B, Campbell SC, Cho HY, Jacqmin D, Lee JE, Weikert S and Kiemeney LA: The epidemiology of renal cell carcinoma. Eur Urol 60: 615-621, 2011.

3. Gudbjartsson T, Hardarson S, Petursdottir V, Thoroddsen A, Magnusson J and Einarsson GV: Histological subtyping and nuclear grading of renal cell carcinoma and their implications for survival: A retrospective nation-wide study of 629 patients. Eur Urol 48: 593-600, 2005.

4. Parkin DM, Bray F, Ferlay J and Pisani P: Global cancer statistics, 2002. CA Cancer J Clin 55: 74-108, 2005.

5. Han S, Wang T, Jiang D, Yu Y, Wang Y, Yan W, Xu W, Cheng M, Zhou W and Xiao J: Surgery and survival outcomes of 30 patients with neurological deficit due to clear cell renal cell carcinoma spinal metastases. Eur Spine J 24: 1786-1791, 2015.

6. Almeida M, Han L, Martin-Millan M, O'Brien CA and Manolagas SC: Oxidative stress antagonizes Wnt signaling in osteoblast precursors by diverting beta-catenin from $\mathrm{T}$ cell factor-to Forkhead Box O-mediated transcription. J Biol Chem 282: 27298-27305, 2007.

7. Brenkman AB, van den Broek NJF, de Keizer PLJ, van Gent DC and Burgering BM: The DNA damage repair protein $\mathrm{Ku} 70$ interacts with FOXO4 to coordinate a conserved cellular stress response. FASEB J 24: 4271-4280, 2010.

8. Roesch A, Mueller AM, Stempfl T, Moehle C, Landthaler M and Vogt T: RBP2-H1/JARID1B is a transcriptional regulator with a tumor suppressive potential in melanoma cells. Int J Cancer 122: 1047-1057, 2008.

9. Katoh M and Katoh M: Human FOX gene family (Review). Int J Oncol 25: 1495-1500, 2004

10. Lu X, Wan F, Zhang H, Shi G and Ye D: ITGA2B and ITGA8 are predictive of prognosis in clear cell renal cell carcinoma patients. Tumour Biol 37: 253-262, 2016.

11. Paner GP, Stadler WM, Hansel DE, Montironi R, Lin DW and Amin MB: Updates in the eighth edition of the tumor-node-metastasis staging classification for urologic cancers. Eur Urol: pii: S0302-2838, 2018.

12. Reddy OL, Cates JM, Gellert LL, Crist HS, Yang Z, Yamashita H, Taylor JA III, Smith JA Jr, Chang SS, Cookson MS, et al: Loss of FOXA1 drives sexually dimorphic changes in Urothelial differentiation and is an independent predictor of poor prognosis in bladder cancer. Am J Pathol 185: 1385-1395, 2015.

13. Guo W, Keener AL, Jing Y, Cai L, Ai J, Zhang J, Fisher AL, Fu G and Wang Z: FOXA1 modulates EAF2 regulation of AR transcriptional activity, cell proliferation and migration in prostate cancer cells. Prostate 75: 976-987, 2015.

14. Zheng L, Qian B, Tian D, Tang T, Wan S, Wang L, Zhu L and Geng X: FOXA1 positively regulates gene expression by changing gene methylation status in human breast cancer MCF-7 cells. Int J Clin Exp Pathol 8: 96-106, 2015.

15. Dou C, Wang Y, Li C, Liu Z, Jia Y, Li Q, Yang W, Yao Y, Liu Q and Tu K: MicroRNA-212 suppresses tumor growth of human hepatocellular carcinoma by targeting FOXA1. Oncotarget 6: 13216-13228, 2015.

16. Yang YA and Yu J: Current perspectives on FOXA1 regulation of androgen receptor signaling and prostate cancer. Genes Dis 2 : 144-151, 2015.

17. Gosalia N, Yang R, Kerschner JL and Harris A: FOXA2 regulates a network of genes involved in critical functions of human intestinal epithelial cells. Physiol Genomics 47: 290-297, 2015.

18. Perez-Balaguer A, Ortiz-Martínez F, García-Martínez A, Pomares-Navarro C, Lerma E and Peiró G: FOXA2 mRNA expression is associated with relapse in patients with triple-negative/basal-like breast carcinoma. Breast Cancer Res Treat 153: 465-474, 2015.

19. Zhu C, Wang J, Shi B, Hu P, Ning B, Zhang Q, Chen F, Chen WS, Zhang $\mathrm{X}$ and $\mathrm{Xie}$ WF: The transcription factor FOXA2 suppresses gastric tumorigenesis in vitro and in vivo. Digest Dis Sci 60: 109-117, 2015. 
20. Zhao YF, Zhao JY, Yue H, Hu KS, Shen H, Guo ZG and Su XJ: FOXD1 promotes breast cancer proliferation and chemotherapeutic drug resistance by targeting p27. Biochem Biophys Res Commun 456: 232-237, 2015.

21. Nakayama S, Soejima K, Yasuda H, Yoda S, Satomi R, Ikemura S, Terai H, Sato T, Yamaguchi N, Hamamoto J, et al: FOXD1 expression is associated with poor prognosis in non-small cell lung cancer. Anticancer Res 35: 261-268, 2015.

22. Van der Heul-Nieuwenhuijsen L, Dits NF and Jenster G: Gene expression of forkhead transcription factors in the normal and diseased human prostate. BJU Int 103: 1574-1580, 2009.

23. JiZ, Donaldson IJ,Liu J, Hayes A, Zeef LA and Sharrocks AD: The forkhead transcription factor FOXK2 promotes AP-1-mediated transcriptional regulation. Mol Cell Biol 32: 385-398, 2012.

24. Zhang G, He P, Gaedcke J, Ghadimi BM, Ried T, Yfantis HG, Lee DH, Hanna N, Alexander HR and Hussain SP: FOXL1, a novel candidate tumor suppressor, inhibits tumor aggressiveness and predicts outcome in human pancreatic cancer. Cancer Res 73: 5416-5425, 2013.

25. Robertson E, Perry C, Doherty R and Madhusudan S: Transcriptomic profiling of Forkhead box transcription factors in adult glioblastoma multiforme. Cancer Genomics Proteomics 12: 103-112, 2015.
26. Qin L, Wu YL, Toneff MJ, Li D, Liao L, Gao X, Bane FT, Tien JC, Xu Y, Feng Z, et al: NCOA1 directly targets M-CSF1 expression to promote breast cancer metastasis. Cancer Res 74: 3477-3488, 2014.

27. De Coo RF, Buddiger P, Smeets HJ and van Oost BA: Molecular cloning and characterization of the human mitochondrial NADH: Oxidoreductase 10-kDa gene (NDUFV3). Genomics 45: 434-437, 1997.

28. Kim JC, Kim SY, Roh SA, Cho DH, Kim DD, Kim JH and Kim YS: Gene expression profiling: Canonical molecular changes and clinicopathological features in sporadic colorectal cancers. World J Gastroenterol 43: 6662-6672, 2008.

29. Park-Hah JO, Kanno H, Kim WD and Fujii H: A novel homozygous mutation of PKLR gene in a pyruvate-kinase-deficient korean family. Acta Haematol 113: 208-211, 2005.

30. Li P, Wong YN, Jahnke J, Pettit AR and Doshi JA: Association of high cost sharing and targeted therapy initiation among elderly Medicare patients with metastatic renal cell carcinoma. Cancer Med 1: 75-86, 2018.

31. Hakimi AA, Reznik E, Lee $\mathrm{CH}$, Creighton CJ, Brannon AR, Luna A, Aksoy BA, Liu EM, Shen R, Lee W, et al: An integrated metabolic atlas of clear cell renal cell carcinoma. Cancer Cell 1: 104-116, 2016. 